\title{
Civilisations
}

Revue internationale d'anthropologie et de sciences

humaines

59-2 | 2011

Les apparences de l'homme

\section{L'ars plumaria en Amazonie}

Pour une esthétique minoritaire

\section{Bertrand Prévost}

\section{(2) OpenEdition}

12 Journals

Édition électronique

URL : http://journals.openedition.org/civilisations/2612

DOI : $10.4000 /$ civilisations.2612

ISSN : 2032-0442

Éditeur

Institut de sociologie de l'Université Libre de Bruxelles

\section{Édition imprimée}

Date de publication : 30 juin 2011

Pagination : 87-108

ISBN : 2-87263-034-1

ISSN : 0009-8140

\section{Référence électronique}

Bertrand Prévost, "L'ars plumaria en Amazonie », Civilisations [En ligne], 59-2 | 2011, mis en ligne le 04 juillet 2014, consulté le 10 décembre 2020. URL : http://journals.openedition.org/civilisations/2612 ; DOI : https://doi.org/10.4000/civilisations.2612

(c) Tous droits réservés 


\title{
L'ars plumaria en Amazonie \\ Pour une esthétique minoritaire
}

\author{
Bertrand PRÉVOST
}

Résumé : Les arts de la plume en Amazonie ont beau passer pour une caractéristique essentielle des cultures des Basses-terres, et au-delà, de toutes les civilisations amérindiennes, ils restent presque totalement délaissés par l'anthropologie. Notre hypothèse est que le désintérêt pour ces objets n'est pas que d'ordre empirique (le manque de faits ethnographiques positifs), mais symptomatise un manquement tant à leur fonction ornementale qu'à leur singularité esthétique. Qu'est-ce qu'une esthétique proprement "plumassière "? Nos modèles occidentaux fondés sur la distinction de la forme et de la couleur, de la surface et du volume, sur un primat de l'inscription (l'opposition figure-fond), ne nous permettent pas de comprendre la singularité d'une parure en plumes. De fait, l'enjeu de cette contribution est de constituer l'unité même de son objet, par-delà la simple unité du matériau.

Mots-clés : plumasserie, Amazonie, parure, esthétique, cosmétique.

\begin{abstract}
Amazon feather-art is well recognized as a main characteristic of the low-lands cultures, and beyond, of all amerindian civilization ; but it remains almost totally left out by anthropology. Our hypothesis is that the disinterest in these objects is not only empirical (the lack of ethnographic data) but reveals a failure both to their ornamental function and aesthetic singularity. What is a proper feather-art? Our western aesthetic models, based on the distinction of form and colour, of surface and volume, on the primate of inscription (the figure-background opposition) make it impossible to understand the singularity of a feather-adornment. So the issue of this contribution is to set up the very unity of its object, beyond the mere unity of the material.
\end{abstract}

Keywords: feather art, Amazon, body ornament, æsthetics, cosmetics. 


\section{$\mathrm{M}$} algré la richesse incomparable de leurs couleurs et de leurs formes, les objets que les Indiens d'Amazonie fabriquent en plume sont des objets de peu. Peu montrés dans les vitrines de nos modernes musées ethnographiques (l'exposition à la lumière pouvant à la longue détériorer l'éclat des couleurs) ou abandonnés dans les réserves à l'appétit des mites, ces objets ont de la difficulté, en Occident, à trouver leur place. Cette visibilité en souffrance n'est évidemment pas de notre seul ressort. En Occident comme en Amazonie, ces objets sont extrêmement fragiles et toujours plus ou moins voués à la disparition. La plume est en effet un matériau organique très délicat, et le seul port de ces parures suffit parfois à les abîmer. De fait, malgré les grands soins portés à leur rangement et leur conservation, les Indiens se voient contraints d'en fabriquer constamment de nouveaux.

Cela n'est dans le fond qu'une contrainte empirique, en soi peu importante. Ce qui l'est beaucoup plus en revanche, c'est que tout se passe comme si la fragilité de ces objets extraordinaires ne concernait pas tant leur existence matérielle que leur existence tout court, que leur statut d'objet à voir, à comprendre, à étudier. Il en irait avec la plumasserie comme autant d'objets légers, sans véritable poids ethnographique, épistémologique et même esthétique. Le parallèle plus ou moins inconscient avec nos propres «trucs en plume » de danseuses de cabaret n'étant pas du meilleur effet pour donner du sérieux théorique et de la consistance esthétique à ces objets. La plume, un matériau déclassé ?

On n'échappera pas à ce fait : l'ars plumaria amazonien est pratiquement laissé pour compte par l'ethnologie. Bibliographie générale extrêmement clairsemée (à quelques très rares exceptions notables), études de terrain parcellaires et aux données très sporadiques, réflexion esthétique totalement inexistante... Les objets en plume seraient des objets de peu de savoir. Cette lacune, dans le fond, n'est pas étrangère à la place particulièrement congrue que l'ethnologie a longtemps réservée aux questions artistiques et plus globalement à ce qu'il est convenu d'appeler « la culture matérielle », cette ethnologie préférant de loin l'étude de l'organisation sociale et des formations symboliques. Au-delà de la difficulté à penser un objet plastique, cette défiance vis-à-vis des objets tient peut-être aux prétentions de rigueur scientifique qu'une discipline aura voulu invoquer, manière pour elle de se distinguer d'un rapport aux objets superficiellement « collectionniste », fétichisant l'objet comme dans un cabinet de curiosité.

Cette lacune tend certes à se combler, mais le discours sur les arts amazoniens demeure souvent empreint d'une généralité inconsistante. Ce n'est certainement pas en tentant de considérer la spécificité d'un objet «art» générique en Amazonie que l'on parviendra à comprendre les objets d'arts singuliers. On ne se défait pas du sentiment que la généralité du discours sur les arts amazoniens a pour pendant le manque de précision et la faiblesse dans l'analyse des objets concrets. Très souvent en effet, "l'approche spécifiquement ethnologique [...] se traduit sur un fond d'interférences commun emprunté, vague et indifférencié : application des grands poncifs, milieu culturel, fonction, valeur à un matériel artistique précis » (Schoepf 1971: 16). Outre la peur que suscite toujours, en ethnologie comme ailleurs, un regard formel ou formaliste sur ces objets, c'est leur nature même qui empêcherait de penser leur singularité esthétique. Car ils participent tous, fondamentalement, des arts de la parure et de l'ornementation corporelle : coiffes, colliers, brassards, bracelets, boucles d'oreille, ceintures... (en considérant qu'ils peuvent également orner des objets : armes, instruments de musique, ustensiles...). À force de ressasser l'évidence selon laquelle 
les objets en plume s'inscrivent dans des usages sociaux, on a tôt fait d'y voir des objets de peu d'art, des objets décoratifs. Tout se passe comme s'il fallait conjurer la dimension ornementale de la plumasserie, au prétexte que la parure serait pour elle-même " purement» esthétique, «simplement» décorative. Mais jamais l'esthétique n'est pure, ni la décoration une chose simple. On voit que cette attitude reconduit plus ou moins inconsciemment tout un système des Beaux-Arts qui privilégiera toujours la statue africaine à la coiffe ou au brassard amazoniens. De la même manière, c'est tout un système philosophique qui se réactualise ici, en niant toute réalité et toute problématicité à l'apparence.

Il appert pourtant que la question de l'apparence n'est pas une mince affaire en Amazonie. Mieux, elle préside à la production même des corps. Les travaux décisifs d'Eduardo Viveiros de Castro $^{1}$, relayés en France notamment par Anne-Christine Taylor (Taylor et E. Viveiros de Castro 2006 : 148-199, Taylor 2010 : 40-51) et Philippe Descola (Descola 2005), ont définitivement achevé le modèle nature-culture, qui avait jusque-là donné tout son sens à la parure humaine conçue comme culturation d'une forme corporelle naturelle. On sait qu'en Amazonie, c'est plutôt l'inverse exact qui se produit, ce que la notion de « multinaturalisme » tend à énoncer, à savoir : tous les êtres partageant une même humanité ("culturelle»), comment penser leur distinction corporelle («naturelle ») ? C'est que la forme n'est en ces terres jamais substantielle, mais toujours relationnelle, ou plus précisément : forme d'un point de vue - entre deux affins, entre un prédateur et sa proie, entre la proie et son prédateur, etc. C'est un vaste perspectivisme (plus qu'une intentionnalité) qui préside ainsi à la production des corps, puisqu'avoir un corps, c'est toujours exister selon un point de vue.

Aussi subtile soit-elle, cette anthropologie de l'apparence ne dit pourtant rien de la singularité de l'ars plumaria. Une coiffe en plumes d'ara revient-elle à la même chose qu'un collier en dents de jaguar? De fait, la question demeure de savoir si la plume peut être élevée au rang d'un matériau esthétique singulier, ou bien si elle occupe une place régulière dans un ensemble plus vaste de matières cosmétiques (perles de verre, graines, os, nacre, élytres, dents, griffes, fibres végétales, poils...). L'un des enjeux de cette étude sera précisément de constituer cette unité esthétique de l'art de la plume, dans son devenir amazonien, puisqu'il s'avère vite que la plume n'est pas une matière comme une autre.

\section{La plume, matière de la différence}

Un mythe parcourt en effet toute l'Amazonie, et se rencontre même au-delà, en Amérique du Nord a ouvert. Il s'agit du mythe dit de l'origine de la couleur des oiseaux ${ }^{2}$. Il connaît de très nombreuses variantes, mais toujours une même structure se déploie : un monstre cannibale, souvent un immense serpent, parfois un aigle harpie géant, sème la terreur parmi les hommes et/ou les oiseaux dont ils sont les alliés. Les oiseaux se liguent et décident d'en finir avec le monstre. Une fois ce dernier vaincu, ils se partagent sa peau multicolore en guise de trophée, chacun revendiquant un morceau. De la sorte, les oiseaux, qui étaient indifférenciés à cette époque, se spécifient en arborant chacun une ou plusieurs couleurs distinctives. Les aras ont par exemple droit à un grand morceau, ce qui explique que leur plumage soit lui-

1. Voir E. Viveiros de Castro 1998 : 469-488 et 2009, notamment : 13-42. Rappelons que la première présentation de cette thèse remonte à l'article de A. Seeger, R. DaMatta, E. Viveiros de Castro (1979 : 2-19).

2. Claude Lévi-Strauss a ouvert ce dossier à la fin de Le cru et le cuit (1964: 308-324). Voir encore le travail de compilation assez décevant de J. F. Pressman (1991: 78-91). 
même multicolore. Les derniers arrivés pour le grand partage demeurent blancs ou noirs. Une variante très répandue explique encore comment le serpent est mortellement blessé (percé par l'assaut des coups de becs) et que les oiseaux trempent alors leur plumage dans son sang multicolore s'écoulant en grandes flaques.

Quelles que soient les variantes, ce mythe énonce toujours un acte de division ou de distinction originaire, une discrimination qui parfois même s'étend au-delà des oiseaux. Toujours est-il que ce sont les oiseaux et leur plumage éclatant qui vont donner matière mythologique et figurale - pour penser la distinction spécifique. C'est que nulle autre matière (poils, végétaux...) que la plume ne se prête mieux à la distinction, en sorte que la plume fournira une sorte de matériau structural de la différence. Daniel Schoepf, dont on ne fait ici que développer l'intuition première, a remarquablement saisi cette nature profondément discriminante de la plume. Pourquoi avoir choisi la parure des oiseaux pour exprimer la différence spécifique ? D'abord parce « la classe des oiseaux est à la fois la mieux circonscrite et celle dont les espèces présentent la diversité d'apparence la plus manifeste » (Schoepf 1985 : 26). La mieux circonscrite : il n'y a que les oiseaux qui ont des plumes. Or, les animaux à poils, à cornes, à écailles ou à carapace ne présentent pas la même unité d'aspect que les oiseaux. Cela nomme une différence spécifique, en soi : les oiseaux vs. les non-oiseaux. Mais la livrée des oiseaux se remarque encore pour ses qualités discriminantes à l'intérieur même de la classe aviaire, mettant en œuvre une différence interne, qui joue à plusieurs niveaux. À un niveau générique ${ }^{3}$, d'abord : tel oiseau aura des plumes noires (le hocco par exemple), tandis que tel autre aura des plumes blanches (le héron par exemple). Mais, le plus souvent à un niveau spécifique également : il est par exemple très aisé de distinguer entre les quatre espèces d'aras dont les Indiens utilisent les plumes, puisque cette distinction se remarque essentiellement par les couleurs du plumage et leur disposition. Mais ces distinctions s'affinent encore à l'échelle individuelle par des différences morphologiques, selon une distribution singulière sur l'oiseau. Si les plumes de l'ara macao sont de loin les plus utilisées dans les basses terres, c'est bien évidemment pour la richesse et l'éclat exceptionnels de leurs couleurs ; mais c'est aussi parce qu'elles se caractérisent autant par une extrême variété que par un caractère distinctif sans équivoque : la forme et la couleur d'une plume permettent immédiatement de la situer sur le corps de l'oiseau. Les Kayapo peuvent ainsi distinguer pas moins de onze types de plumes sur le seul corps de l'Ara macao ${ }^{4}$.

C'est cette nature éminemment différenciée qui donne à la plume toute son efficace différenciante. "C'est donc dire que la plume est par excellence un matériau d'ordre, un matériau qui ordonne, et ceci en plus de toutes ses qualités de diversité chromatique. La plume implique la logique d'un classement par catégorie et par espèce » (Schoepf 1985 : 26). Matière de la différence : c'est par elle que les divisions sociales s'exprimeront avec le plus de force et de lisibilité. Cela se vérifie par exemple dans l'usage quasi nul que les Kayapo font des rémiges d'ara (à l'exception, justement, du marché pour touristes) : puisque les quatre espèces d'aras ont toutes les ailes bleues (avec des petites différences chromatiques certes), et ne se distinguent, faiblement, que par la couleur de leur revers. Plus généralement, c'est ce souci de différenciation qui permet sans doute d'expliquer le faible usage que les Indiens

3. « Générique » et « spécifique » (cf. infra) sont ici utilisés au sens strict de la taxinomie.

4. Voir G. Verswijver (1995: 351), pour le détail de la nomenclature. 
font des plumes vertes, puisqu'elles se rencontrent sur un très grand nombre de perroquets et de perruches, et prêteraient donc à la confusion.

La plumasserie kayapo est tout à fait exceptionnelle sur ce point. En effet, la complexité de l'organisation sociale de ce vaste groupe est ici reflétée par la richesse et la variété de ses parures, principalement en plumes. On sait que le port des parures dépend chez ces Indiens de critères circonstanciels (usage quotidien ou lié à un rituel particulier) et catégoriels (usage déterminé par le sexe, la classe d'âge ou la position rituelle) que viennent recouper des critères prérogatifs, autrement dit des privilèges personnels donnant droit à user d'un certain type de plumes pour un certain type de parures (parmi d'autres droits). Ces privilèges dépendent du segment que l'on occupe dans le village (le segment est toujours déterminé par le lieu de naissance de la mère) et se transmettent individuellement. En effet, les maisons d'un village kayapo s'ordonnent traditionnellement en un cercle, découpé en un certain nombre de segments occupés chacun par une famille uxorilocale. Et cette division obéit encore à un principe cosmique relatif à la course du soleil. Bref, il en ressort une très grande complexité sociologique qui trouve sa matière première ou expressive ${ }^{5}$ dans la plume. La variété intrinsèque des plumes, redoublée par la variété des types de parure est telle qu'elles peuvent remplir la totalité des cases du tableau par lequel l'ethnologue aura systématisé l'ensemble de ces divisions sans répétition ni zones indistinctes ${ }^{6}$.

\section{Une élégance souveraine}

Élever la plume au rang d'un matériau théorique exprimant à merveille les subtilités des divisions sociales et cosmiques nous fait certes avancer, mais rate encore la particularité de l'ars plumaria en tant qu'art ornemental. C'est qu'une véritable anthropologie esthétique ne peut faire l'économie de l'efficacité formelle, en sorte que le schème forme-matière s'en trouvera nécessairement critiqué. Il reste à tenir le point de vue d'une morphologie générale de la parure, qui fait cruellement défaut en anthropologie, aussi bien d'ailleurs que dans tous les champs censés rendre compte de la chose artistique.

On ne pourra faire l'impasse sur l'élégance souveraine qui anime ces objets. Cette élégance ne saurait tenir à leur seul degré de perfectionnement technique, ni à leur finition exceptionnelle. Elle dépend plus profondément de choix esthétiques, dont il faut, au moins à grands traits, tenter de repérer les grandes lignes. On doit à Georg Simmel, dans un texte aussi bref qu'efficace, d'avoir lancé quelques pistes pour mieux comprendre la logique formelle de ce que l'on nomme parure. Une parure est toujours un ornement corporel qui entretient un rapport morphologique au corps plus ou moins adéquat : elle colle plus ou moins à sa forme. Or, c'est dans la modulation de ce rapport que la parure tire toute son efficacité esthétique : entre la forme de l'ornement et la forme (de la partie) du corps orné.

Tout ce qui, de façon générale, "pare " l'être humain, prend place sur une échelle de degrés, selon la façon plus ou moins étroite dont la parure est liée à la personnalité physique. La parure la plus étroitement liée au corps est caractéristique des peuplades primitives : c'est le tatouage. L'extrême opposé, c'est la parure de métal ou de pierre, qui n'est absolument pas liée à un individu et que chacun peut mettre. Entre les deux, il

5. Puisque le système des parures est autant l'expression d'un ordre social (préexistant) que sa justification ou sa cause même (il faut diviser le collectif pour distribuer les parures).

6. Sur tout cela, voir les travaux décisifs de G. Verswijver 1982-1983 : 23-62 et 1992 : 65-87. 
y a le vêtement - qui certes n'est pas aussi inéchangeable et personnel que le tatouage, mais qui est tout de même plus lié à l'individu et moins détachable de lui que la 'parure' à proprement parler. Mais c'est précisément dans l'impersonnalité de celle-ci que réside son élégance. Alors que la pierre et le métal sont entièrement clos sur eux-mêmes, ne désignant aucune individualité précise et non modifiables du fait de leur dureté, ils sont malgré tout forcés de servir une personnalité : là est précisément l'attrait le plus subtil de la parure. L'élégance véritable évite l'individualisation extrême, elle instaure toujours une sphère de généralité, de stylisation, pour ainsi dire d'abstraction autour de l'individu [...]. (Simmel $1998:$ 82-83)

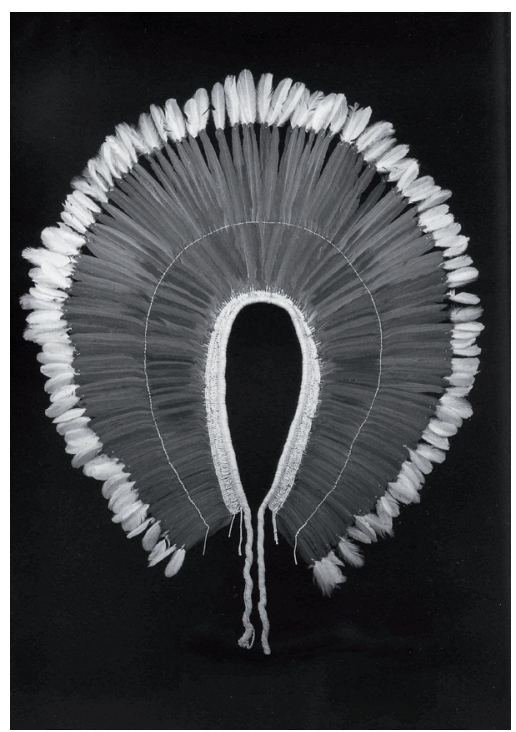

Figure 1

Parure dorsale (krokrokti), Kayapo, plumes et coton.

Tervuren, Musée Royal de l'Afrique Centrale, D.R.

Qu'est-ce que Simmel entend ici par « impersonnalité » de la parure, sinon le mouvement d'une décorporation? Parler de «parure abstraite» signifie alors que celle-ci n'entre dans aucune relation mimétique ou d'imitation formelle avec le corps orné. Mieux : elle gagne en élégance ce qu'elle perd en ressemblance avec ce qu'elle pare. Cette abstraction de la parure dit son autonomie corporelle, son inadéquation organique. Jean-Clet Martin a largement insisté sur ce «charme incorporel» de la parure qui ouvre les contours organiques d'un corps, et l'expose parfois jusqu'à l'extase (Martin 2003). Abstraite du point de vue de la forme organique, la parure ne manque pourtant pas, pour elle-même, d'individualité. Au contraire : sa souveraine élégance tient précisément à sa très grande précision, à sa distinction formelle - individuelle - tout à fait remarquable en Amazonie, par-delà le seul fini technique.

En un mot, l'ars plumaria nous offre le modèle idéal pour penser la nature anorganique de telles parures : ni totalement inorganique, puisqu'elle est précisément faite de plumes ; ni totalement organique, puisqu'elle demeure inadéquate à la forme corporelle qu'elle orne. Cette désorganisation doit donc se mesurer dans un double rapport : au corps paré 


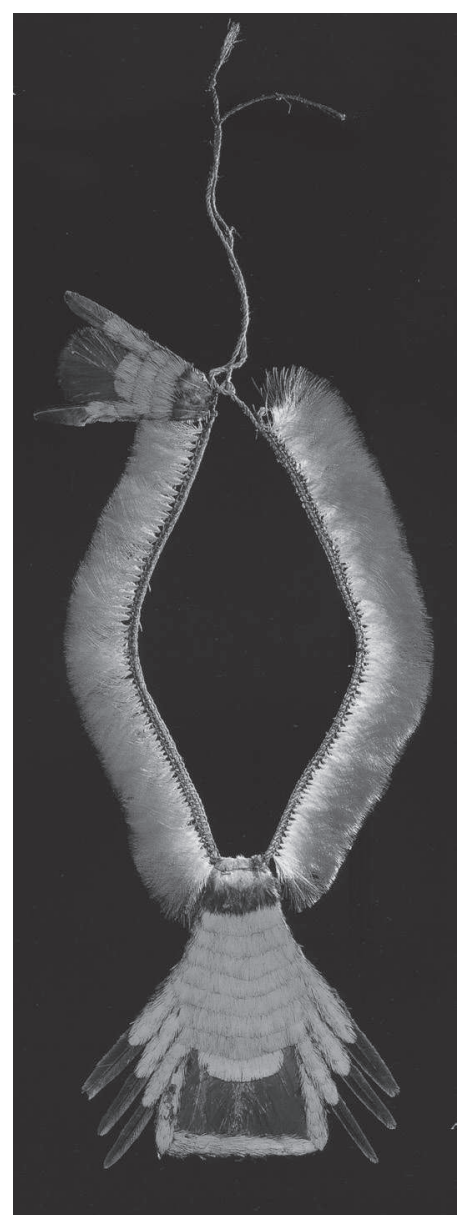

Figure 2

Collier, Urubu-Ka'apor, plumes, fibre végétale, coton, colle. Coll. part., D.R.

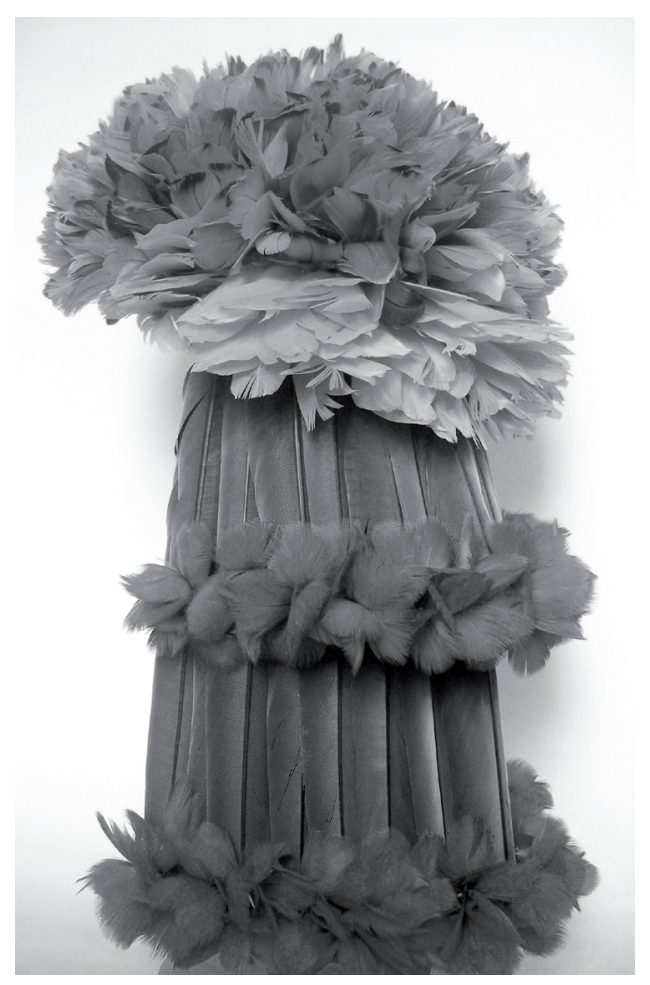

Figure 3

Coiffe couvre-nuque, Mundurucu, plumes, coton, fibre végétale, colle. Coll. part., D.R. 
(la forme humaine) mais également, voire d'abord, au corps « parant», si l'on peut dire (la forme animale). Reprenons. La « désorganisation » de la forme aviaire en passera par une transformation du matériau organique lui-même : la plume. À l'exception des plumules ou plumes de corps, une plume se caractérise par une morphologie essentiellement longitudinale (elle est toujours plus longue que large). Or, il appert que la technique d'assemblage la plus fréquente en Amazonie consiste précisément à inverser cette morphologie pour produire une surface de plumes plus large que longue. Il s'agit du montage dit en filière : chaque plume est attachée en repliant sa hampe sur un fil d'appui, tandis qu'un second fil vient ligaturer le repli par un noeud, un peu plus haut sur la hampe. En sorte que c'est bien une étendue superficielle qui se construit, et qui contrevient à la nature radiante qui caractérise la forme et la disposition des plumes sur l'oiseau (la longue queue des aras pousse cette morphologie à son comble). Il est ainsi significatif que les diadèmes rayonnants soient relativement rares en Amazonie (à l'exemple du pariko bororo), les Indiens préférant un montage des plumes en filière nettement plus serré (voir par exemple le krokrokti des Kayapo, pourtant fait avec des pennes caudales d'ara, qui cherche au maximum à faire surface plutôt que rayon; cf. fig. 1). Cette «désorganisation» tient encore à des transformations texturales. L'autre caractère morphologique de la plume tient à sa structure superficielle, puisqu'elle a (entre autres) une fonction de couverture (par une lointaine transformation des écailles). Il faut voir alors comment les Urubu-Kaapor, par une petite modification du montage traditionnel en filière, transforment radicalement cette structure plane en une structure pileuse, où chaque plume devient plutôt une touffe. C'est là un trait remarquable de leur plumasserie, qui contribue à sa préciosité et à sa minutie, et qui se rencontre sur presque tous les bracelets, brassards, colliers et ceintures (fig. 2). Néanmoins, la transformation la plus spectaculaire vient sans doute du rôle éminemment plastique conféré à la plume et qui contrevient manifestement à cette structure superficielle que nous venons de décrire. Or, les parures en plume ont toujours une voluminosité singulière, qui ne tient que rarement à la forme d'un support, mais qui est le plus souvent construite ou produite, et qui ne tire sa consistance que des plumes elles-mêmes. La plumasserie mundurucu est sur ce point tout à fait remarquable ${ }^{7}$ (fig. 3), elle qui fait un grand usage du montage en touffe et en pétale. Le couvre-nuque des coiffes, notamment, fait de deux filières superposées (l'une dépassant l'autre de moitié) de plumes caudales d'ara, à l'extrémité desquelles plusieurs touffes de plumes ont été montées en pétale (il s'agit le plus souvent de plumes noires de hocco, plus rarement d'ara), est une pure construction plumassière qui se déploie plastiquement et qui de fait ne connaît plus aucune distinction entre structure et décoration.

À l'autre pôle, c'est bien la forme corporelle humaine qui est « abstraite », qui voit son organisation se défaire. Prenons le cas des couvre-chefs ${ }^{8}$. On pourrait instituer une échelle d'adhésion formelle, exprimant la plus ou moins grande adéquation entre la parure et la forme de la tête. Le premier type serait occupé par les bonnets emplumés : roriro-rê et kraimrojakati des Kayapo, lori-lori des Karaja (fig. 4), bonnets des Mundurucu (fig. 3). Ici, la parure est encore très proche de la forme ovoïde qui caractérise le crâne humain, et peut parfois (dans le cas de certains bonnets kayapo) reprendre la disposition en écaille des plumes

7. Sur la plumasserie mundurucu, voir D. Campana (1905 : 177-197).

8. Il est entendu que les limites de cette contribution ne nous permettent pas de dresser une morphologie générale. 
sur le corps de l'oiseau ainsi que la distribution des couleurs. Les diadèmes, couronnes et bandeaux (la tawashap jivaro, les nombreux diadèmes faits d'un cerceau de palme sur lequel sont fixées des plumules d'Ara ou de Toucan, fréquents dans les Guyanes, chez les Wayapi, les Waiwai ou les Wayana, toutes les couronnes rayonnantes montées sur un support rigide ; fig. 5) présentent eux aussi une dépendance forte vis-à-vis du pourtour crânien. Les tiares communes dans la région du Xingu (entre autres chez les Kamayura, les Mehinaku, les Yawalapiti ; fig. 6), en revanche, franchissent peut-être une étape dans cette morphologie de « l'impersonnalité physique », pour reprendre la terminologie de Simmel, dans leur capacité à faire se dresser une crête. Il y a pourtant encore un rapport certain avec l'idée d'intériorité ou de boîte, qu'annulent définitivement les petites coiffes akka des Kayapo (fig. 7), dont l'usage est presque quotidien. Ces dernières constituent une véritable protubérance sur la tête, qui, si elle demeure dans un voisinage formel avec le cercle de la tête, ne s'en détachent pas moins à la manière d'une crête (l'ondulation dans la partie centrale supérieure, quand la coiffe est portée, venant encore perturber la planéité du nimbe).

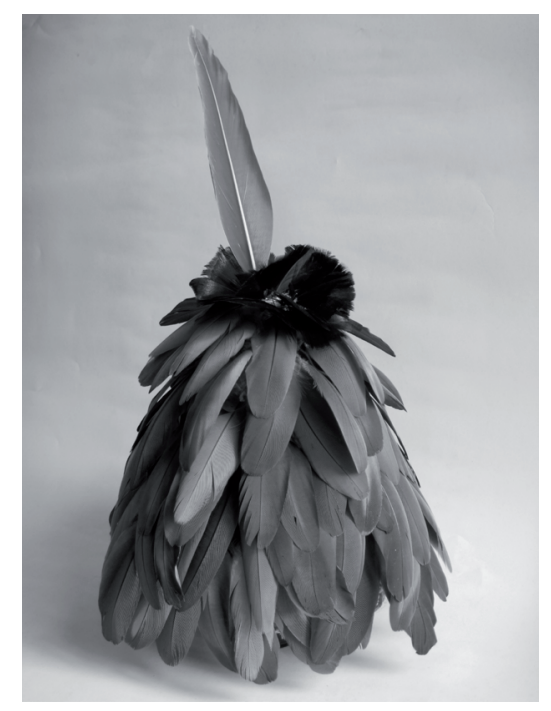

Figure 4

Chapeau (roriro-ri), Kapayo, plumes, coton, fibre végétale. Coll. part., D.R.

Les parures les plus abstraites ou les plus « impersonnelles » dans les termes de Simmel, vont donc être celles qui rompent tout schéma de prolongement, d'étirement ou d'extension du corps. Il resterait à interroger la notion de protubérance : excroissance, décollement, détachement d'une forme d'un ensemble organisé. Il en irait de toutes ces plumes plantées dans le corps : les ornements nasaux rikbaktsa (deux plumes fichées en travers des narines ; fig. 8) ; les labrets urubu-kaapor (une protubérance labiale formant comme une grosse langue de plume) ; ou encore d'un étrange ornement dorsal kayapo : une (plus rarement plusieurs) plume qui se dresse (beaucoup plus qu'elle ne pend) à partir du cou (tout sauf un collier) et qui dessine quelque chose comme une improbable queue, comme si l'appendice caudal 
avait remonté jusqu'à la tête. Ces parures sont relativement locales et ne se rattachent qu'à une partie assez peu étendue du corps humain, d'où leur capacité à se désolidariser plus facilement de la forme corporelle globale. De ce point de vue, la grande parure kayapo, akkati-rê (fig. 9), tire sa suprême élégance de sa profonde étrangeté, dans la mesure où le détachement ou le décollement du corps s'y entend littéralement, alors même que l'on a affaire à une grande parure globale ou englobante : une grande filière de plumes montée sur un support rigide (en fibres de palme) en forme de fer à cheval allongé, tenu en équilibre sur la tête, vient dessiner un nimbe autour de la tête, des épaules et du dos. L'impersonnalité de la parure, pour reprendre la terminologie de Simmel, est telle qu'elle ne touche même plus l'individualité physique.

Le formalisme objectiviste de cette typologie non indigène nous exposera fatalement à une condamnation, pour avoir commis le crime de lèse-majesté ethnologique : l'ethnocentrisme. Car il semble que notre interprétation contrevienne à l'idée que l'ethnologie se fait du cosmétique amazonien. Diadèmes en plumes, peintures corporelles, colliers, boucles d'oreilles, labrets, etc., loin de se penser comme autonomes et désolidarisés du corps, devraient davantage être conçus comme une extension ou une expansion du corps. Ainsi, chez les Kayapo et dans de nombreux autres groupes, les distensions de la bouche et des oreilles produites par les plateaux labiaux et les boucles d'oreilles sont interprétées analogiquement comme des formes d'amplification des facultés de communication sociale : parole et écoute9. Les parures en plumes se prêteraient aisément au même type d'interprétation puisqu'elles tendent toutes peu ou prou à élargir les limites corporelles voire à agir comme un nimbe somptueusement coloré émanant de la personne.

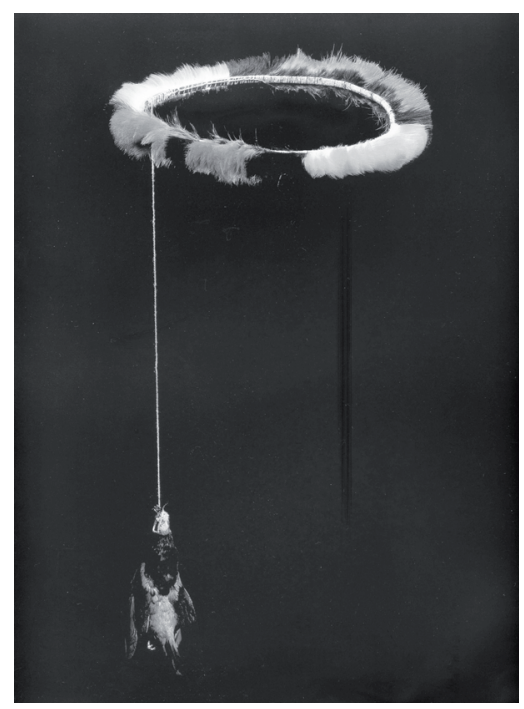

Figure 5

Diadème, Wayapi, plumes, dépouille d'oiseau, fibre végétale, coton, colle. Sao Paulo, Museu de Arqueologia e Etnologia da Universidade de Sao Paulo, D.R. 


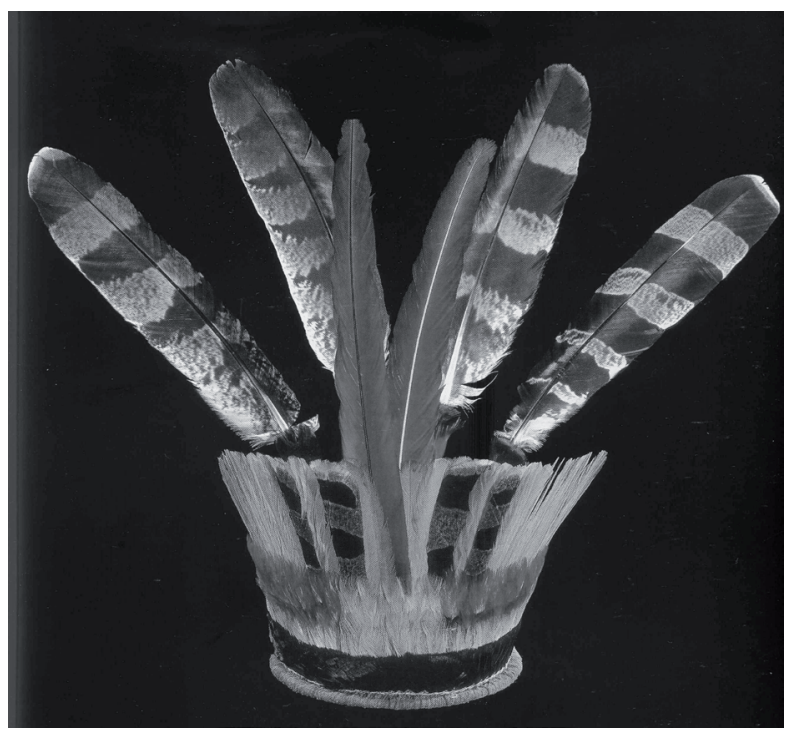

Figure 6

Tiare (tukanap), Kalapalo, plumes, fibre végétale, coton. Coll. part., D.R.

Il n'empêche que cette vision de la parure comme extériorisation esthétique d'une subjectivité intérieure, comme extension d'une individualité semble bien davantage reconduire à notre très classique notion de décoration comme « beauté adhérente » (le decordecus des Anciens) plutôt qu'à la conception proprement amazonienne de la corporéité. Et c'est évidemment ici que l'on retrouve le perspectivisme et sa force critique. En effet, si la subjectivité s'entend en Amazonie comme quelque chose qui s'endosse, être sujet, c'est toujours occuper un corps vu par d'autres corps, comme on revêt un habit pour occuper une position. Changer de corps par sa parure, c'est donc changer de perspective, occuper un autre point de vue. Le schème perspectiviste suppose ainsi l'idée d'une corporéité amovible ou à disposition, cette disponibilité disant précisément la variabilité et l'extériorité des points de vue possibles ${ }^{10}$. Or, ce qu'on a reconnu comme la «nature anorganique » des parures en plumes se prête admirablement à cette idée de corps amovible, désolidarisé de toute forme d'intériorité formelle. Du reste, on comprend parfaitement l'association des plumes avec toutes ces autres matières anorganiques : cheveux, poils, écailles, piquants, que presque toute l'Amazonie pense comme un même ensemble. La langue témoigne d'ailleurs souvent de l'usage d'un même mot pour le désigner. C'est bien la position intermédiaire de

10. Philippe Descola souligne donc avec raison l'inadéquation ou l'hétérogénéité formelle que l'animisme installe entre l'intériorité et le corps, c'est-à-dire entre un corps nu ou neutre (échappant aux regards) et un corps à l'apparence singulière (pris dans un point de vue) : «Dans une ontologie marquée par la séparabilité de l'âme et du vêtement corporel, on n'est jamais sûr de l'identité réelle de la personne qui se cache à l'intérieur de l'enveloppe que l'on perçoit. Un humain peut s'incorporer dans un animal ou une plante, un animal adopter la forme d'un autre animal, une plante ou un animal ôter son habit pour mettre à nu son intériorité objectivé dans un corps d'humain » (Descola $2010: 25$ ). 
ces matériaux qui leur confère leur puissance de subjectivation : ni totalement extérieurs (puisqu'ils poussent à partir du corps dont ils sont une production), ni totalement intérieurs (puisqu'ils tombent, muent et se détachent du corps). Non que la plume ne soit pas corporelle, elle n'est que cela au contraire ! Mais c'est qu'elle contrevient à toute idée d'opposition entre corps propre (le Leib allemand) et corps physique (Körper) et oblige à penser un «corps impropre », impersonnel mais néanmoins singulier.

À l'extrême rigueur, la notion de «point de vue», malgré sa très grande portée philosophique, fait encore courir le risque d'assigner la subjectivité à un point, fût-il de vue, et d'empêcher de comprendre que la subjectivité s'entend plutôt en Amazonie comme une affaire de surface. Le fait que le nom générique pour désigner les parures en plume soit celui de " peau » est suffisamment éloquent (pour ne rien dire évidemment du rapport originel des corporéités spécifiques avec la peau du monstre primordial) ${ }^{11}$. Mais, pour reprendre le célèbre mot de Valéry, « la peau est ce qu'il y a de plus profond », façon de dire que cette peau, cette matière superficielle porte en elle-même sa subjectivité, ou plutôt, elle est matière subjectivante, perspective : « peau de vue » plus que « point de vue », osera-t-on dire ${ }^{12}$.

\section{Grands écarts, petites différences (Leibniz sous les tropiques)}

Nous progressons, mais il reste encore à comprendre que ce déficit en savoir dont pâtit la plumasserie amazonienne symptomatise un plus fondamental déficit en voir, par delà l'économie de sa visibilité. Dit autrement, les arts de la plume ne sont pas laissés dans l'ombre pour de seules raisons matérielles et épistémologiques, mais peut-être d'abord pour des raisons esthétiques. Il faudrait rendre de compte de ce statut de l'ars plumaria que l'on dira esthétiquement minoritaire ${ }^{13}$. Non pas du tout mineur parce qu'il ressortirait aux arts décoratifs de la parure corporelle, mais bien esthétiquement minoritaire, dans toute sa singularité formelle et technique.

Cette minorité esthétique n'est sans doute pas étrangère au soubassement mythologique que nous avons précédemment rappelé. Les mythes sur l'origine des couleurs des oiseaux sont des mythes de la distinction, qui tous mettent en œuvre un dynamisme de partition ou de partage. L'ars plumaria amazonien ne fera que prolonger dans l'ordre esthétique ce travail de la différence. De fait, presque toutes les parures en plume se remarquent par leurs très grands écarts, (au moins) chromatiques. Les ruptures de tons sont extrêmement franches et comme les couleurs des plumes sont en elles-mêmes particulièrement vives et en un nombre relativement restreint, l'effet contrastif n'en est que plus marqué. C'est globalement que les unités sont appréhendées, comme de véritables blocs de couleur.

11. Exemple kayapo, parmi tant d'autres : toutes les parures formées sur le mot $a k k a$ («peau [d'oiseau]») : akka-ti ("grande peau d'oiseau »), akkakry-re («petite peau d'oiseau »), akkakre, akkapa-ri,... Pour une étude très approfondie de toute la plumasserie kayapo, voir G. Verswijver 1995 : 77-164.

12. A contrario, il est significatif que Terence Turner voie dans la peau une « frontière de l'individu », comme surface d'intercession entre la constitution biologique de l'individu et une projection sociale, en rejouant donc l'opposition classique nature/culture. Voir i.a. Turner (1977 : 162-171, article de 1969).

13. Cela dit en référence à la notion de « littérature mineure » développé par Gilles Deleuze et Félix Guattari à propos de Kafka (Deleuze et Guattari 1975). 
Il n'empêche que cet état de fait ne résiste pas à l'effectivité esthétique et technique des parures en plume. C'est que ces grands écarts chromatiques viennent très souvent se doubler de très petits écarts, aussi bien chromatiques que formels. Pris dans leur distinction relative (différence spécifique), les ensembles colorés sont parfaitement distincts, mais ils sont pour eux-mêmes parcourus par une multitude de petites distinctions ou de petites différences (différence en soi), de telle sorte que c'est la partie elle-même qui continue de se diviser, non plus de façon claire et distincte mais sur un mode presque imperceptible. Ce travail de la nuance est évidemment très remarquable quand on a affaire à une parure monochrome. Prise comme unité, chaque plume a la même taille, la même forme, la même couleur, éventuellement le même motif. Mais pour elle-même, aucune plume n'est exactement identique à une autre : il y a toujours au minimum une petite modulation d'une plume à l'autre, d'autant plus marquée que les plumes proviennent d'un nombre important d'individus: le jaune d'une plume caudale de japu sera plus doré ici, plus clair là, légèrement tacheté de noir ailleurs... C'est ici qu'il faudrait également faire mention de cette pratique de décoloration naturelle des plumes, le tapirage, qui a longtemps laissés incrédules les ethnologues ${ }^{14}$. Le tapirage permet certes de changer la teinte des plumes sur le corps du perroquet vivant, afin d'obtenir une coloration jaune (couleur somme toute assez rare dans l'avifaune amazonienne, au moins pour les pennes), mais on oublie aussi qu'il provoque presque toujours une modulation chromatique, en introduisant une nuance rouge-orangée à la base du rachis ${ }^{15}$.

Ce jeu des nuances et des petites différences ne tient que partiellement à un état de fait de la matière disponible. Il est évident que les propriétés de la plume tiennent à sa nature de matière naturelle, organique, qui ne peut pas passer sur les petites différences individuelles comme le ferait une production industrielle. Il n'empêche que cela ne représente aucunement une contrainte empirique et extérieure sur l'ars plumaria, mais correspond bien à un choix esthétique et technique tout à fait assumé en Amazonie. Autrement dit, les petites différences sont tout autant recherchées que les grands écarts. En somme, tout se passe comme si ce qui, dans le mythe, était de l'ordre de la division en parties extensives (chaque espèce étant corrélée à une couleur elle-même corrélée à une partie tout à fait localisable du corps du monstre primordial) se transformait dans l'ars plumaria en degrés intensifs.

Cela se vérifie magnifiquement dans «bourrage schizophrénique $»^{16}$ auquel donnent très souvent lieu les parures en plume et qui contribue à porter la matière à sa «loi d'extremum, [à savoir :] un maximum de matière pour un minimum d'étendue ${ }^{17}$. C'est en effet une caractéristique constante de tous ces ornements que d'être bourrés de plumes, que de présenter une densité tout à fait remarquable. Leur fabrication requiert souvent une

14. Voir l'article classique d'Alfred Métraux, « Une découverte biologique des Indiens de l'Amérique du Sud : la décoloration artificielle des plumes sur les oiseaux vivants » (Métraux 1928 : 181-192).

15. Voir également sur Internet la page [anonyme] : http://caiquesite.com/Published\%20articles/tapiragem. htm. Il semblerait que la matière dont les Indiens enduisent la peau du perroquet (sécrétion de peau de grenouille, graisse de poisson, sang...) n'ait pour fonction efficace que de détériorer la structure du follicule (ce qui expliquerait au passage pourquoi le seul fait de plumer régulièrement un oiseau comme le pratiquent les Indiens, par l'irritation produite, suffit à modifier sensiblement, et en partie, la couleur des plumes, sans pouvoir parler de « tapirage » proprement dit).

16. Selon la formule de Gilles Deleuze à propos du tableau baroque (Deleuze 1988 : 166).

17. Ibid. 
très grande quantité de plumes, alors même que la surface à construire ou à recouvrir est relativement peu étendue. Un bonnet mundurucu nécessite par exemple (fig. 3) pratiquement un millier de plumes montées en paires sur une petite calotte de coton crocheté. De même, la grande parure dorsale kayapo, le krokrokti (fig. 1), peut nécessiter jusqu'à trois cents plumes caudales d'ara, pour une longueur d'un peu plus d'un mètre. Ou encore, les petits bracelets arara des Urubu-Kaapor, qui réunissent environ soixante-quinze plumes de duvet d'ara pour une longueur d'à peine plus de dix centimètres. Techniquement, cette densité tient très souvent à un montage des plumes en filière particulièrement resserré. Or, il se trouve que là où quelques plumes ou quelques dizaines de plumes (selon la longueur de la filière à emplumer) suffiraient à produire une continuité, l'emplumage est toujours extrêmement serré, en sorte que toutes les plumes se chevauchent les unes les autres. Cette imbrication des plumes est telle qu'elle ne laisse souvent même pas apparaître leur rachi. Ce n'est donc absolument pas l'économie de matière première qui préside à la fabrication de tels objets, mais bien le contraire : il s'agit de charger de plume au maximum. L'effet qui en résulte tend à donner à la filière, pourtant plate et superficielle, une plasticité voire une voluminosité qui ne tient qu'à la seule quantité de plumes utilisées. Une appréciation « esthétique », comme l'on dit ${ }^{18}$, au détour d'un mythe kaapor sur l'origine des parures, confirme la validité esthétique de ce montage imbriqué, puisque le narrateur parle des anciennes parures en plumes, « plus belles que celles d'aujourd'hui » comme des parures « grandes et épaisses » (Ribeiro 2002: 486). Que vient faire ici l'épaisseur, dans un type de parure (l'akangatar, fig. 10), ne consistant qu'en la superposition de filières de plumes cousues sur une bande de coton, sinon souligner la recherche du bourrage et de la densité ?

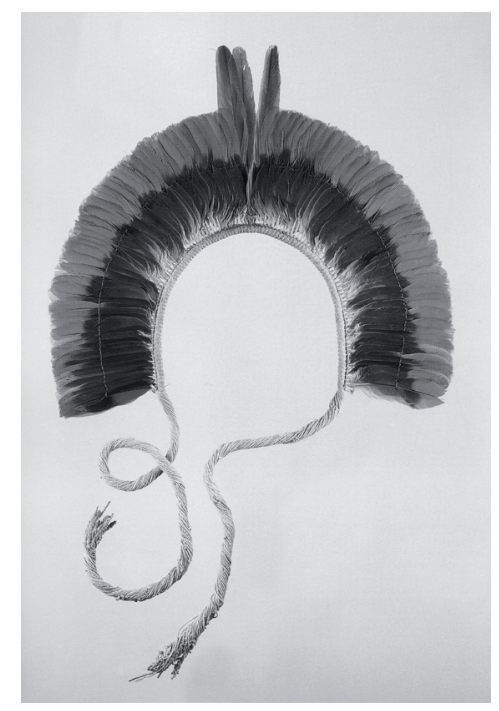

Figure 7

Coiffe (akkakê-rê), Kayapo, plumes, coton.

Sao Paulo, Museu de Arqueologia e Etnologia da Universidade de Sao Paulo, D.R.

18. À supposer que l'esthétique soit question de jugement et d'appréciation, ce que nous ne croyons pas du tout. 


\section{Ars plumaria et peinture}

Faire de l'ars plumaria un art esthétiquement minoritaire, ne tient pas, du moins pas exclusivement, aux seules propriétés formelles ni à l'usage d'un matériau singulier. Si l'ars plumaria est un art minoritaire, c'est qu'il entre dans un rapport critique très étrange avec les catégories plastiques traditionnelles - majoritaires - de l'art occidental, à commencer par la peinture. Il ne s'agit pourtant pas simplement de dire que ce devenir-minoritaire tient à son exclusion vis-à-vis de ces expressions artistiques : un " art inclassable » pour nous autres Occidentaux. Il faut plutôt montrer qu'une confrontation s'établit, que sans être réductible à de la peinture, il y a devenir-minoritaire précisément parce que s'engage un rapport critique avec ce mode majeur ${ }^{19}$.

Si les arts amazoniens commencent à être considérés, c'est au prix d'un déséquilibre manifeste, révélant autant une erreur ethnologique qu'un symptôme esthétique. C'est que cette considération se focalise essentiellement sur les peintures corporelles, les tatouages, la poterie voire la vannerie. Cette exclusion, répétons-le, fait symptôme, et par conséquent doit être interprétée pour elle-même. Toutes ces formes d'expression artistique - à l'exception de la vannerie ${ }^{20}$ - ont en commun de penser la forme d'un unique point de vue graphique, où ce qui fait figure est toujours tracé ou dessiné. Il est tout à fait symptomatique à cet égard que, malgré ses mises en garde contre tout ethnocentrisme, Marcel Mauss, justement à propos de l'ornementique, ait reconduit un tel point de vue, en affirmant que « l'art le plus élémentaire de la plastique est évidemment le dessin graphique, qui, même quand il porte sur un volume est toujours réductible à un dessin en plan $»^{21}$. Mais d'où l'ethnologue tient-il que «l'art le plus élémentaire de la plastique est évidemment le dessin graphique »? - Il le tient du primat que l'Occident aura toujours reconnu au modèle projectif, du mythe d'origine de la peinture selon Pline l'Ancien, où la fille du potier Dibutades circonscrit sur un mur l'ombre projetée du profil de son amant avant son départ (Pline l'Ancien XXXV : 15), à l'expérience inaugurale de Brunelleschi devant le Baptistère de Florence, « démontrant » la perspective par une projection en miroir ${ }^{22}$, jusqu'à la théorie classique du disegno (dessein-dessin) comme projet. Autrement dit, d'un modèle qui ne conçoit l'avènement d'une forme que comme la projection d'une figure sur un fond, que comme l'application d'un motif sur un plan.

Il ne s'agit donc pas d'arguer que l'ars plumaria serait d'abord un art de la couleur, en retombant dans les affres du vieux débat, toujours mal posé, entre le dessin et la couleur. Bien au contraire. On osera dire que l'ars plumaria amazonien est autant un art de la couleur que de la figure, mais sur un mode qui n'a rien de projectif ${ }^{23}$. Les parures présentent certes des couleurs éclatantes mais elles n'en sont pas pour autant a-figurales. Figuratives, elles ne sont que très rarement : les colliers féminins urubu-kaapor sont peut-être la seule exception, en Amazonie (fig. 2). Mais figurales, elles le sont tout le temps : coiffes, brassards ou colliers ne

19. Deleuze et Guattari soulignaient bien qu'« une littérature mineure n'est pas celle d'une langue mineure, plutôt celle qu'une minorité fait dans une langue majeure », cf. Deleuze et Guattari 1975 : 29.

20. Ce qui laisse peut-être préjuger d'un rapport avec l'ars plumaria, ce qui se confirmera infra.

21. M. Mauss $1967: 102-103$ (nous soulignons).

22. Voir sur ce point les travaux décisifs d'Hubert Damisch (1997).

23. On s'étonnera par exemple de ce que Peter G. Roe fasse de l'ars plumaria un « art graphique », voir P. G. Roe $1990: 105$. 
sont pas des touts indifférenciés (on a montré tout le contraire !), et présentent toujours des distinctions formelles tout à fait marquées, au minimum sous la forme de bandes abstraites régulières, produites par la superposition de filières (fig. 5, 6, 7, 9, 10) ou par les motifs naturellement présents sur la plume (fig. 7). Tout cela dessine bien des motifs abstraits, géométriques le plus souvent, qui tiennent leur stabilité figurale autant de leur forme que de leur couleur. On comprend du reste l'avantage du montage serré des plumes en filière : cela permet justement la constitution de ces motifs, qui perdraient leur distinction ou leur précision figurales par un nombre trop faible de leurs constituants. C'est de ce point de vue qu'un rapport avec la peinture - la nôtre - s'établit. Mais s'il y a «peinture de plumes » en Amazonie ce n'est absolument pas sur le mode projectif qui a prévalu en Occident (au moins jusqu'au milieu du $\mathrm{XX}^{\mathrm{e}}$ siècle). Tout le problème est ici : il y a figure sans qu'il y ait projection.

Cette question ne ressortit pas à l'ars plumaria en général, mais bien à son devenir amazonien. Car rien n'interdit de faire un usage projectif de la plume ${ }^{24}$. Et de fait, la « peinture de plumes » a bien existé dans l'Amérique pré- et post-colombienne, où " peinture » se sera entendu en un sens majeur ou majoritaire, comme l'inscription d'une figure (colorée) sur un fond. Nous faisons par exemple allusion à ces extraordinaires textiles emplumés du Pérou ancien (Nazca Huari) : tapisseries, ponchos, capes, chapeaux et autres bijoux (Reid 2005) ... Dans tous ces cas, cousues ou collées, les plumes fonctionnent comme une matière d'inscription sur un support. Il en va de même avec les célèbres mosaïques de plume que les plumassiers aztèques ont su pratiquer avec tant d'adresse, et dont il ne reste pratiquement rien. Il s'agissait de coller sur un support végétal durci une infinité de plumules, le plus souvent coupées à leur extrémité pour obtenir un motif figuratif ou géométrique extrêmement précis. On sait le succès que cet art a rencontré dans le Mexique post-colonial, mais aussi dans toute l'Europe moderne, sous la forme de ces «tableaux de plumes »: ces retables et autres objets liturgiques chrétiens réalisés par les artisans indiens, au moins jusqu'au $\mathrm{XVIII}^{\mathrm{e}}$ siècle $^{25}$. Cet engouement ne tient pas selon nous au seul goût pour les exotica et les hybridation culturelles. Nous voulons dire que les modèles esthétiques européens étaient parfaitement aptes à « digérer » ce type de plumasserie puisqu'il ne remettait absolument pas en cause la prégnance d'un modèle projectif, qui commençait qui plus est à forger ses armes théoriques ${ }^{26}$. C'est peut-être ici qu'une technique mineure montre toute sa dimension politique $^{27}$, puisque cette distinction entre une plumasserie projective et non-projective (on verra plus loin comment lui conférer toute sa positivité) semble corrélative d'une division à la fois politique, entre sociétés à État (moderne ou archaïque) et « sociétés contre l'État ${ }^{28}$,

24. Les exceptions sont extrêmement rares en Amazonie : en plus de certains ornements kaapor, mentionnons des pectoraux wayana et les masques ype (cara grande) des Tapirape.

25. Voir entre autres A. Erlande-Brandeburg, Le triptyque aztèque de la crucifixion, Paris, Rmn, 2004.

26. A. Russo (2009 : 153-164) souligne bien la proximité esthétique et technique qui a été vue dès le XVI ${ }^{\mathrm{e}}$ siècle entre la mosaïque de plumes et la peinture.

27. Sur le rapport entre mineur et politique, voir G. Deleuze et F. Guattari $1975: 30-31$.

28. Pour reprendre une division conceptuelle chère à P. Clastres dans La société contre l'État (1974). 
et idéologique, entre systèmes de pensée analogiques ou naturalistes et systèmes de pensée animistes $^{29}$.

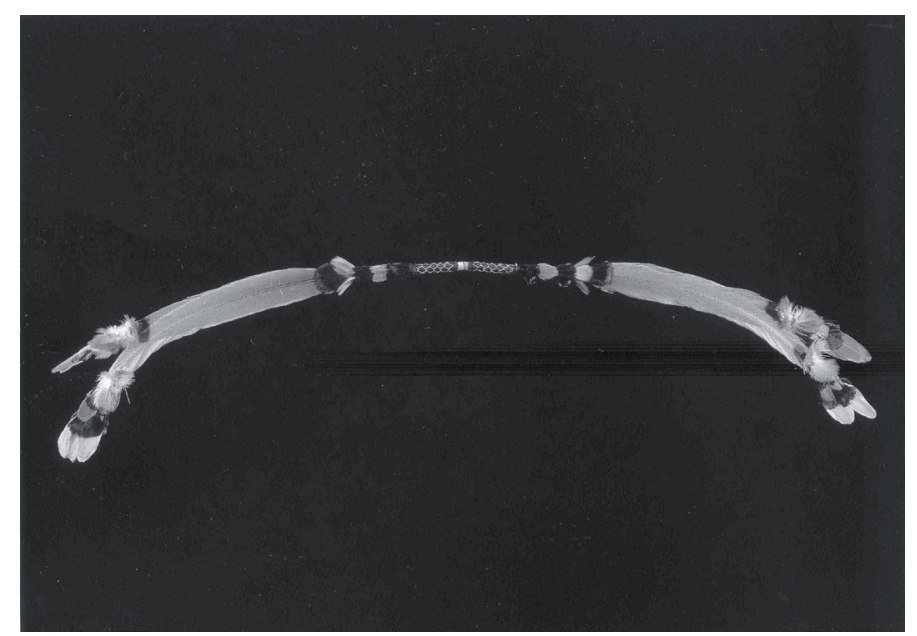

Figure 8

Ornements nasaux, Rikbaktsa, plumes, roseau, fibre végétale, colle. Coll. part., D.R.

\section{Plumes, vannerie, tissage}

On pourra toujours objecter qu'à bien considérer l'ensemble de l'ars plumaria amazonien, les cas abondent où l'ornement corporel en plume non seulement nécessite techniquement une importante structure sur laquelle il va reposer (cousu ou simplement attaché), mais encore affiche esthétiquement cette dernière, en la faisant rentrer dans une complémentarité avec la partie proprement en plume ${ }^{30}$. Les couronnes ou tiares de nombreux groupes du Xingu (fig. 6) tiennent bien par un support rigide en vannerie, qui peut d'ailleurs être partiellement peint, orné de motifs tressés, voire recouvert de fourrure. Plus généralement, la plume est souvent associée avec un élément structurel et/ou décoratif : vannerie, fourrure, perles de verre, peinture, coquillages, fil de coton... Sauf que ce qui officierait alors comme subjectile n'a jamais la neutralité d'un plan d'inscription continu, abstrait ou théorique, puisque tressé, tissé voire perlé, il est toujours déjà figural, formellement différencié : au minimum par le tissage même des fibres, au maximum par l'existence de véritables motifs. On retrouve de fait cette antériorité déjà soulignée du complexe sur le simple, du discontinu sur le continu, du différencié sur l'indifférencié : motif sur motif.

29. Pour reprendre la classification que produit P. Descola dans Par-delà nature et culture (2005).

30. Cela pourrait d'ailleurs recouper la distinction que Berta Ribeiro avait jadis établie pour les couvre-chefs entre les supports flexibles et les supports rigides, les premiers étant tendanciellement destinés à être cachés, les seconds à être délibérément montrés. Voir B. G. Ribeiro $1957: 59-119$. 


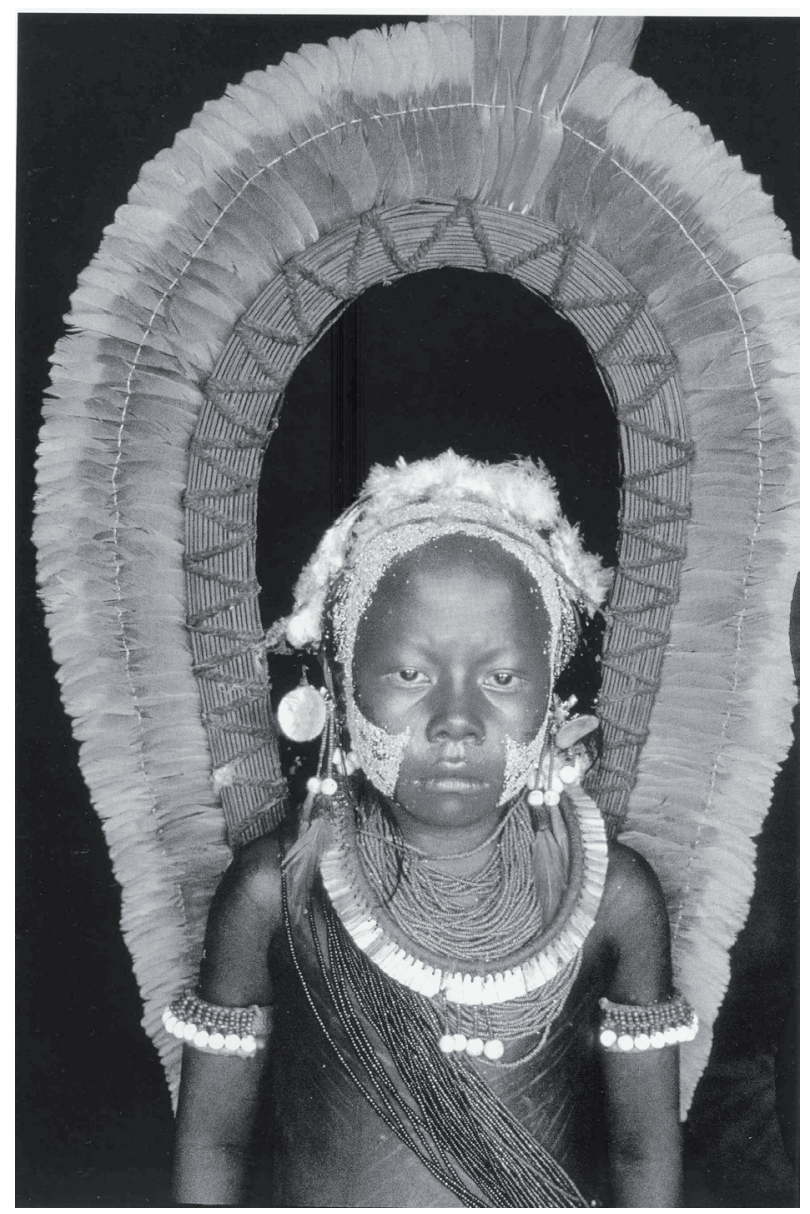

Figure 9

Jeune Kayapo portant une parure dorsale (akkati-rê). Coll. part., D.R.

En Amazonie, le textile ne peut jamais être considéré comme support esthétiquement neutre, car de fait, il n'est presque jamais techniquement support. Il est tout à fait significatif par exemple que les manteaux tupinamba, qui ont fait jadis la richesse des Wunderkammern, ne soient pas tissés mais consistent en une résille uniquement constituée des ligatures des plumes $^{31}$. On pourrait encore mentionner la tawasap des Jivaros, dont la partie textile n'est pas un bandeau tissé mais un filet, dont les brins sont tressés à chaque extrémité ; ou encore les bonnets karaja. Quoi qu'il en soit, les rares exceptions de supports tissés - sous forme notamment de bandeaux, comme avec l'akangatar urubu-kaapor (fig. 10) ou certaines coiffes cashinahua - ne fonctionnent jamais comme plan de projection.

31. Voir A.M. Métraux (1928 : 140-148). Métraux, p. 147, voit parfaitement la différence entre ces manteaux et les « tuniques de plumes » de l'Ancien Pérou où « les plumes sont cousues sur une pièce d'étoffe ». 


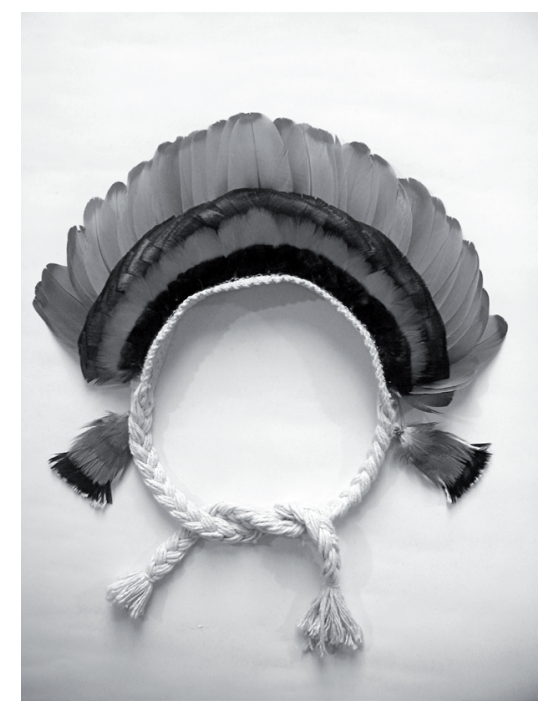

Figure 10

Diadème (akangatar), Urubu-Ka'apor, plumes, coton, colle. Coll. part., D.R.

S'il y a quelque chose de tissé dans l'ars plumaria amazonien, ce n'est pas un support plan, ce sont les plumes elles-mêmes. L'emplumage en filière extrêmement dense et serré dont nous avons fait état pourrait à cet égard fournir un jalon pour apparenter la plumasserie aussi bien au tissage qu'à la vannerie. Le principe technique est, d'un certain point de vue, toujours le même : chaque unité (fil, fibre, plume) est prise entre un dessus et un dessous, dont la capacité à former surface tient à l'agrégation serrée, comprimée ou pressée et non lâche ou détendu (sauf effets de jours recherchés, mais qui doivent être compensés ailleurs pour assurer la tenue du produit). Pour ne rien dire évidemment du montage des plumes lui-même (la filière n'étant qu'un mode technique parmi d'autres, certes les plus répandu) qui ressortit fondamentalement à une technique de ligature et de nouage, le collage étant rarissime (ce sont à l'occasion les fils eux-mêmes qui sont encollés). La proposition que Daniel Schoepf faisait il $\mathrm{y}$ a près de quarante ans déjà nous paraît donc des plus justes : «il ne semble pas prématuré dans l'état de nos connaissances, d'affirmer que les principales manifestations artistiques des Indiens de l'Amazonie ne relèvent ni de la taille ni du modelage, mais bien du tressage et du ligaturage $»$ (Schoepf $1971: 17)$.

\section{Le modèle matriciel : plumes, vannerie, tissage, peinture}

L'apparentement de la vannerie et de l'art textile avec l'ars plumaria ne se limite pas à un rapprochement empirique, fondé sur des effets esthétiques, aussi prégnants soient-ils, et des solutions techniques, aussi élaborées soient-elles. Cet alliance est d'abord transcendantale en ce qu'elle dépend d'un modèle théorique extrêmement profond qui va tresser - si l'on peut dire ! - les brins technique, esthétique et mythologique. À tel point que la peinture elle-même sera intégrée dans ce modèle. Il eût en effet semblé bien étrange que la peinture corporelle 
s'entendît en Amazonie sous le mode dominant qu'elle connaît en Occident ${ }^{32}$. C'est qu'elle ne s'y conçoit jamais comme le recouvrement d'une surface par projection de matière, mais plutôt comme la configuration de motifs déjà donnés.

Il revient à la mythologie d'avoir pensé ce modèle théorique anti-projectif en lui donnant toute sa positivité esthétique, au titre d'un modèle que l'on dira matriciel ${ }^{33}$. Cette matrice est constituée par le corps de ce serpent primordial cannibale que l'on a déjà rencontré. C'est le partage de sa peau multicolore qui donnait aux oiseaux leur couleur. Mais c'est aussi cette même peau qui va donner leurs motifs à la peinture et à la vannerie (le déploiement de complexes figures géométriques rayées ou losangées ou simplement tachetées sur le corps des boïdés ainsi que leur aspect écailleux donnant bien évidemment un substrat organique). Les Wayana expliquent par exemple comment tous les motifs de leur vannerie proviennent de l'entrevue des flancs de Tulupérê, un monstre aquatique tueur d'hommes ${ }^{34}$. Il est très important de remarquer que couleurs et figures sont pensées dans une origine commune, qui donc prête à la couleur une figuralité originelle, à l'opposé de la continuité qualitative par laquelle l'Occident pense le chromatisme.

Il ne s'agit pas seulement de faire de la peau du serpent une sorte de réserve originaire dans laquelle les Indiens viendraient puiser leurs motifs, un catalogue de figures et de couleurs destiné à être copié. N'oublions pas que de nombreux mythes présentaient déjà la coloration des oiseaux comme un bain dans le sang multicolore du serpent. C'est davantage comme corps matriciel qu'il faut envisager le serpent. Et de fait, une matrice n'est pas un stock ni un magasin : c'est un lieu de production, une intériorité productrice qui fonctionne par invagination, plissement, involution..., soit un dynamisme interne, qui suppose et l'intériorité et le mouvement. Or les mythes abondent, qui présentent l'origine des parures, de plumes ou de peinture, comme des histoires où un homme se fait avaler par le serpent : le héros est avalé tout cru et continue de vivre à l'intérieur du monstre. Quand il en ressort, il est paré des plus beaux atours, de plumes et de peinture. Toutes les variantes sont possibles : dans un cas $\left(\right.$ Kaapor $\left.^{35}\right)$, il se déplace dans le corps du serpent pour le tuer de l'intérieur ; dans un autre (Waiwai Fock 1963 : 91-92), il n'est avalé que partiellement, puis recraché, la partie de son corps avalée se voyant décorée de superbes motifs ; ailleurs encore (Macurap B. Mindlin 2005: 60-63), c'est avec ses dents et les caresses de sa langue que l'anaconda peint le corps du héros. À ces variations sur les dynamismes (déplacement, rejet, caresse...) correspondent autant de variations sur l'intériorité, puisque c'est autant la bouche, l'intérieur de tout le corps, le trou du serpent, voire sa maison qui vont donner figure à cette intériorité. Autant dire que ces mythes concernent beaucoup moins le fait d'être mangé que d'être sujet d'une immersion figurale et chromatique, dont résulteront les plus somptueuses parures.

$$
*
$$

32. Nous disons bien dominant, car l'effectivité des œuvres - du moins de certaines -, depuis presque toujours, n'aura pas manqué de critiquer un tel modèle depuis son intérieur même.

33. Il est entendu que les analyses qui suivent n'ont de valeur qu'introductive. Nous nous réservons le droit de revenir ailleurs et plus en profondeur sur la question.

34. Voir L. Hussak van Velthem (1998), notamment 119-131.

35. Voir D. Ribeiro 2002 : 611-613, ce mythe très important met en corrélation l'origine de la voie lactée, l'origine de la couleur des oiseaux et l'origine des parures. 
En articulant esthétique, technique et mythologie, nous n'avons pas tant cherché à identifier les références culturelles de l'ars plumaria qu'à saisir sa consistance singulière : façon de poser quelques jalons pour la description d'une authentique pensée plumassière en Amazonie. C'est moins la pensée indienne d'un phénomène esthétique (génitif objectif) qui nous aura retenu, que la pensée d'un phénomène esthétique indien (génitif subjectif); moins la représentation indigène de l'art de la plume que ce que l'art de la plume fait aux représentations indigènes autant qu'occidentales. L'invocation du contexte culturel ou de la prise en compte du point de vue indigène, en effet, n'éclaire rien tant qu'elle se comprend comme une seule question de référence. Elle peut bien expliquer localement une fonction ou une signification mais ne peut que rater la logique propre d'un phénomène. Il est étonnant de constater que l'on tend encore à refuser aux objets plastiques ce que Lévi-Strauss avait pourtant accordé, et avec quel succès, aux mythes : leur capacité à « se pense[r] entre eux » (Lévi-Strauss 1964 : 20), autrement dit à dessiner une sorte de plan de consistance qui ne connaisse plus l'opposition entre l'objectivité du savant et la subjectivité du point de vue indigène. Il serait bien dommage que l'ethno-esthétique tombe dans les mêmes pièges que ceux où l'histoire de l'art n'a cessé de tomber depuis des décennies, sinon depuis toujours. Devant une parure en plumes comme devant une peinture de la Renaissance, il n'y a pas à séparer, encore moins à opposer la forme (style), le contenu (signification) et la fonction. Tant que l'on fait de l'objet la représentation d'un style conçu comme catégoriel ou d'un ensemble de significations et de fonctions culturelles, autrement dit, tant que l'on considère l'objet comme une matière qui ne trouverait de sens que par l'imposition d'une forme extérieure et antérieure, on passe fatalement à côté de sa singularité : soit sa capacité à s'individuer (comme on parle d'individuation en philosophie), à cristalliser en une forme singulière un ensemble de problèmes esthétiques, sociaux, techniques, mythologiques qui ne semblent pas liés a priori mais qui se nouent et se précipitent sous l'espèce de cette forme plastique.

\section{Références bibliographiques}

Campana, D., 1905. « L'arte plumaria dei Mundurucu (Brasile) e di altri popoli del Sud-America », Archivio per l'antropologia e la etnologia, 35 : 177-197.

Clastres P., 1974. La société contre l'État. Paris : Minuit.

Damisch, H., 1997. L'origine de la perspective. Paris : Champs Flammarion.

Deleuze, G., 1988. Le Pli. Leibniz et le baroque. Paris : Minuit.

Deleuze G. et F. Guattari, 1975. Kafka. Pour une littérature mineure. Paris : Minuit.

Descola, P., 2005. Par-delà nature et culture. Paris : Gallimard.

Erlande-Brandeburg, A., 2004. Le triptyque aztèque de la crucifixion. Paris : Rmn.

Fock, N. , 1963. Waiwai Religion and Society of an Amazonian Tribe. Copenhague : The National Museum.

Hussak van Velthem, L., 1998. A pele de Tuluperê. Uma etnografia dos trançados Wayana. Belem : Museu Paraense Emilio Goeldi.

LÉvi-Strauss, 1964. Le cru et le cuit. Paris : Plon.

Martin, J. C., 2003. Parures d'Eros. Un traité du superficiel. Paris : Kimé.

Mauss, M., 1947. Manuel d'ethnographie. Paris : Payot. 
MÉtraux, A.M., 1928. «Une découverte biologique des Indiens de l’Amérique du Sud : la décoloration artificielle des plumes sur les oiseaux vivants », Journal de la Société des Américanistes, 20 : 181-192.

--_-, 1928. La civilisation matérielle des tribus Tupi-Guarani. Paris : Librairie orientaliste Paul Geutner.

Mindlin, B., 2005. Fricassée de maris. Mythes érotiques de l’Amazonie, trad. J. Thiériot. Paris : Métailié.

Pline l’Ancien, 1947. Histoire Naturelle. Paris : Les Belles Lettres.

Pressman, J. F., 1991. « Feathers of Blood and Fire. The Mythological Origin of Avian Coloration », in R. E. Reina et M. Kesinger (éd.), The Gift of Birds. Featherwork of Native South American People. Philadelphie : The University Museum of Archeology and Anthropology-University of Pensylvania.

Reid J. W., 2005. Magic Feathers. Textile Art from Ancient Peru. Londres : Textile \& Art Publications.

Ribeiro, B. G., 1957. « Bases para uma classificação dos adornos plumários dos 'Indios do Brasil », Arquivos do Museu Nacional, 43 : 59-119.

2002. Carnets indiens. Avec les Indiens Urubus-Kaapor, Brésil, trad. J. Thiériot. Paris.

Roe P. G., 1990. "The Language of the plumes : «Implicit Mythology », in Shipibo, Cashinahua and Waiwai Feather Adornments », in. M. H. Preuss, (éd.), LAIL Speaks! Selected Papers from the VII International Symposium on Latin American Indian Literatures. Culver City : Labyrinthos.

Russo, A., 2009. "Image-plume, temps-reliquaire ? Tangibilités d'une histoire esthétique », in G. Careri, F. Lissarrague, J.-C. Schmitt, C. Severi (éds.), Traditions et temporalités des images. Paris : Éd. de l'EHESS.

SchoеpF, D., 1971. « Essai sur la plumasserie des Indiens Kayapo, Wayana et Urubu - Brésil », Bulletin annuel du musée d'ethnographie de Genève, 14 : 15-68.

1985. « Pourquoi la plume ? Ordre, esthétique et identité dans les cultures amérindiennes », in D. Schoepf (éd.), L'art de la plume. Brésil. Genève : Musée d'ethnographie.

Seeger A., 1975. « The Meaning of Body Ornaments : A Suya Example », Ethnology, (14) 3 : 211-224.

Seeger A., R. DaMatta, et E. Viveiros de Castro, 1979. «A construção da pessoa nas sociedades indígenas brasileiras », Boletim do Museu Nacional, 32 : 2-19.

Simmel G. 1998. « Psychologie de la parure », in La parure et autres essais. Paris : Éditions de la MSH.

TAYLOR, A. C., 2010. «Voir comme un autre : figurations amazoniennes de l'âme et des corps », in P. Descola (dir.), La Fabrique des images. Paris : Musée du quai Branly-Somogy : 40-51.

Taylor, A. C. et E. Viveiros de Castro, 2006. «Un corps fait de regards », in S. Breton (dir.), Qu'est-ce qu'un corps? Paris : Musée du quai Branly-Flammarion : 148-199.

Turner, T., 1977. «Cosmetics: The language of bodily adornment», J. P. Spradley \& D.W. McCurdy (éds) Conformity and Conflit. Readings in cultural Anthropology. Boston, Toronto : Little, Brown \& Compagny, 162-171 (article de 1969).

1995. « Social Body and Embodied Subject : Bodiliness, Subjectivity and Sociality among the Kayapo », Cultural Anthropology, 10 (2) : 143-170.

Verswijver, G., 1982-1983. «Essai sur l'usage de la parure chez les Indiens Kaiapó du Brésil Central », Bulletin annuel du Musée d'ethnographie de la Ville de Genève, 25-26 : 23-62

1992. « Only you may wear my ornament », in G. Verswijver (éd.), Kaiapó. The Art of Body Decoration, Gand : Musée Royal de Tervuren, Snoeck-Ducaju \& Zoon : 65-87.

1995. Kaiapo. Materielle Kultur, spirituelle Welt / Material Culture, Spiritual World. Frankfort s/Main : Museum für Völkerkunde : 77-164.

Viveiros de Castro, E., 1998. "Cosmological Deixis and Amerindian Perspectivism », Journal of the Royal Anthropological Insistute (N. S.), (4) $3:$ 469-488.

2009. Métaphysiques cannibales. Lignes d'anthropologie post-structurale, trad. O. Bonilla. Paris : PUF. 


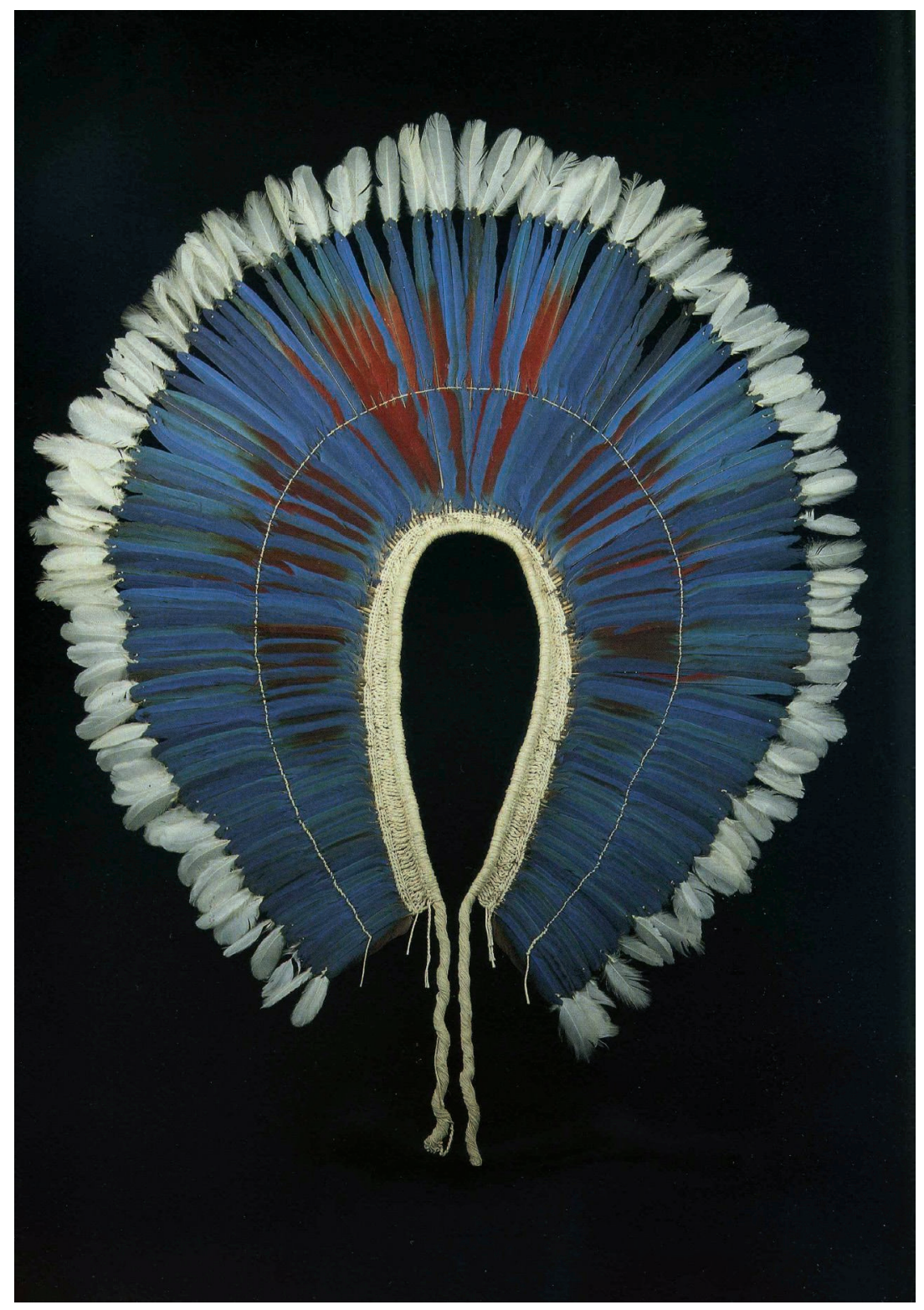

\section{Cf. Prévost, figure 1}

Parure dorsale (krokrokti), Kayapo, plumes et coton. Tervuren, Musée Royal de l'Afrique Centrale, D.R. 


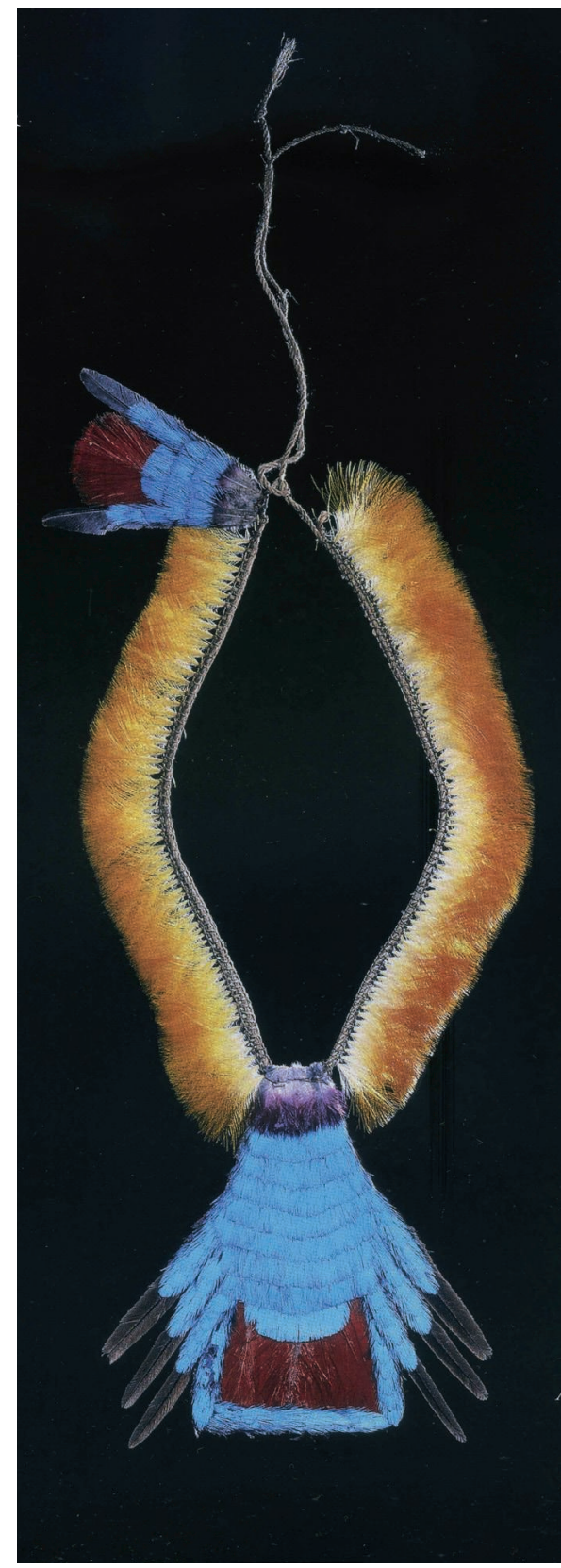

Cf. Prévost, figure 2

Collier, Urubu-Ka'apor, plumes, fibre végétale, coton. Coll. part., D.R. 


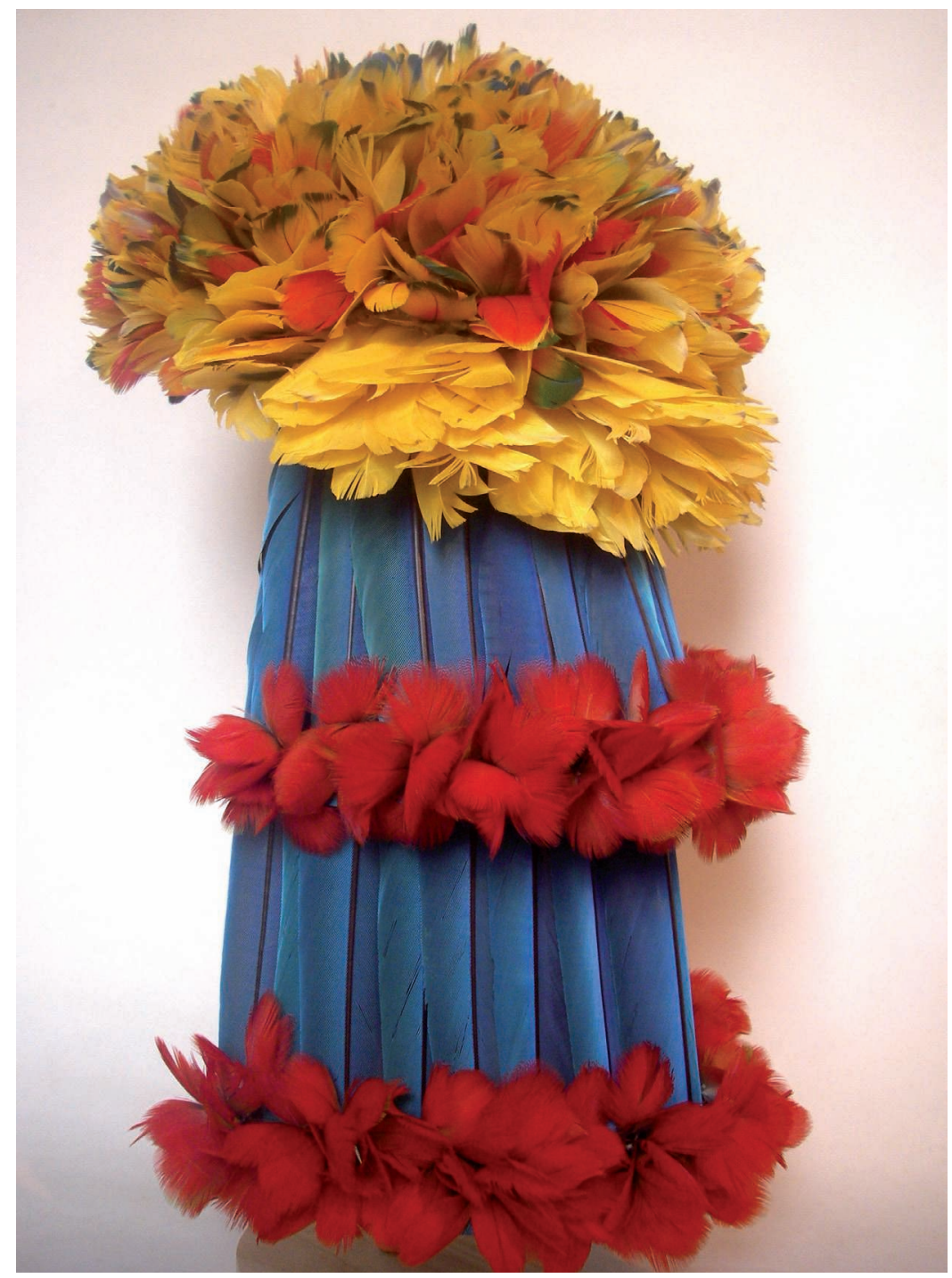

\section{Cf. Prévost, figure 3}

Coiffe couvre-nuque, Mundurucu, plumes, coton, fibre végétale, coton. Coll. part., D.R. 


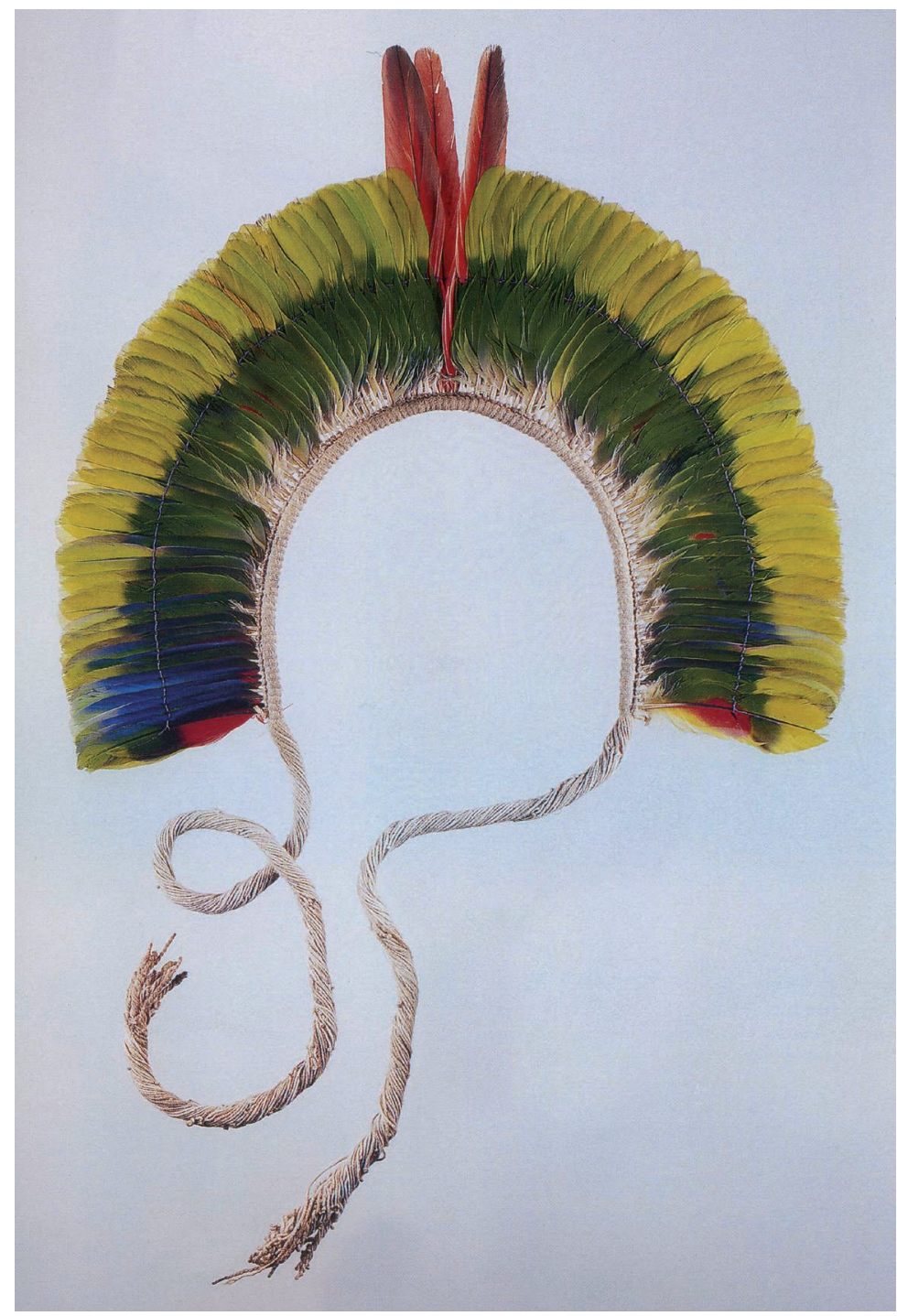

Cf. Prévost, figure 7

Diadème (akangatar), Urubu-Ka'apor, plumes, coton, colle. Coll. part., D.R. 


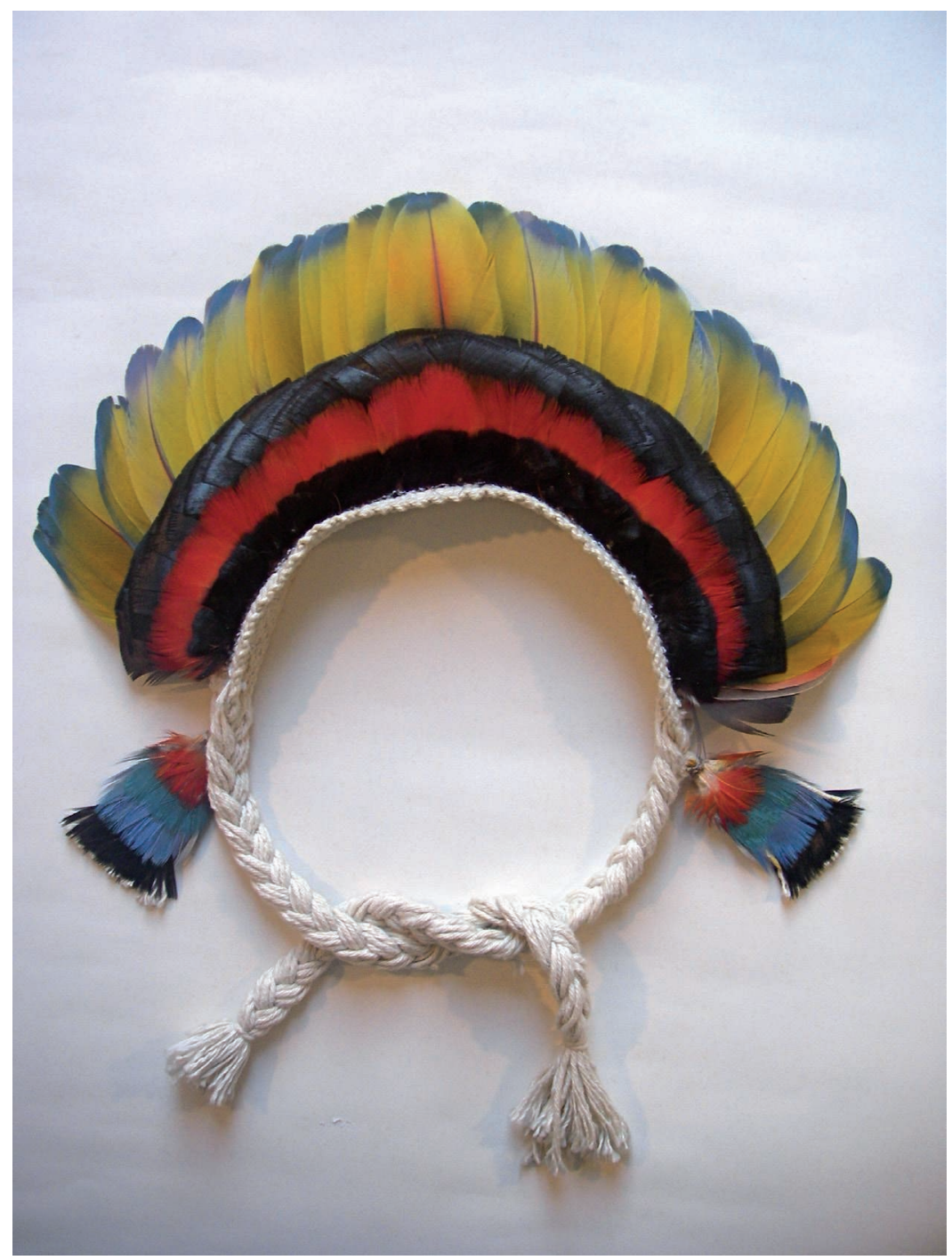

Cf. Prévost, figure 10

Diadème (akangatar), Urubu-Ka'apor, plumes, coton, colle. Coll. part., D.R. 